\title{
PERIODIC SOLUTIONS IN A DELAYED PREDATOR-PREY MODEL WITH NONMONOTONIC FUNCTIONAL RESPONSE
}

\author{
LIN-LIN WANG, YONG-HONG FAN AND WEI-GAO GE
}

\begin{abstract}
By using the continuation theorem of coincidence degree theory, the existence of a positive periodic solution for a delayed predator-prey model with nonmonotonic functional response

$$
\left\{\begin{array}{l}
x^{\prime}(t)=x(t)(a(t)-b(t) x(t))-(x(t) y(t)) /\left(m^{2}+c x(t)+x^{2}(t)\right), \\
\left.y^{\prime}(t)=y(t)(\mu(t) x(t-\tau)) /\left(m^{2}+c x(t-\tau)+x^{2}(t-\tau)\right)-d(t)\right)
\end{array}\right.
$$

is established, where $a(t), b(t), \mu(t)$ and $d(t)$ are all positive periodic continuous functions with period $\omega>0, c>0, m>0$ and $\tau$ is a nonnegative constant. In particular, our result improves one former conclusion.
\end{abstract}

1. Introduction. In microbial dynamics or chemical kinetics, the functional response describes the uptake of substrate by the microorganisms. In general the response function $f(x)$ is monotone. However, there are experiments that indicate that nonmonotonic responses occur at the microbial level: when the nutrient concentration reaches a high level an inhibitory effect on the specific growth rate may occur. This is often seen when microoganisms are used for waste decomposition or for water purification, see Bush and Cook [3]. The so-called Monod-Haldane function

$$
f(x)=\frac{c x}{m^{2}+b x+x^{2}}
$$

has been proposed and used to model the inhibitory effect at high concentrations, see Andrews [1]. In experiments on the uptake of

2000 AMS Mathematics subject classification. Primary 34K15, 92D25, 34C25.

Keywords and phrases. Predator-prey model, nonmonotonic functional response, positive periodic solution, coincidence degree.

Research supported by the Natural Science Foundation of Ludong University (24070301, 24070302, 24200301), Program for Innovative Research Team in Ludong University and China Postdoctoral Science Foundation funded project (20080430314). The second author is the corresponding author.

Received by the editors on June 5, 2007, and in revised form on January 2, 2008.

DOI:10.1216/RMJ-2008-38-5-1705 Copyright (c)2008 Rocky Mountain Mathematics Consortium 
phenol by pure culture of Pseudomonas putida growing on phenol in continuous culture, Sokol and Howell [14] proposed a simplified MonodHaldane function of the form

$$
f(x)=\frac{c x}{m^{2}+x^{2}}
$$

and found that it fits their experimental data significantly better than the Monod-Haldane function and is simpler since it involves only two parameters.

Ruan and Xiao [12] studied the system with this simplified MonodHaldane functional response:

$$
\left\{\begin{array}{l}
x^{\prime}(t)=r x(t)[1-(x(t) / K)]-(x(t) y(t)) /\left(m^{2}+x^{2}(t)\right), \\
y^{\prime}(t)=y(t)\left[(\mu x(t)) /\left(m^{2}+x^{2}(t)\right)-d\right] .
\end{array}\right.
$$

And, for the standard Holling type IV function, i.e., the MonodHaldane function, Zhu, Campbell and Wolkowicz [19] gave a detailed analysis of the system

$$
\left\{\begin{array}{l}
x^{\prime}(t)=r x(t)[1-(x(t) / K)]-(x(t) y(t)) /\left(a x^{2}(t)+b x(t)+1\right), \\
y^{\prime}(t)=y(t)\left[(\mu x(t)) /\left(a x^{2}(t)+b x(t)+1\right)-d\right] .
\end{array}\right.
$$

Based on some experimental data, Caperson [14] observed that there is a time delay between the changes in substrate concentration and the corresponding changes in the bacterial growth rate. Following Caperson's observation, Bush and Cook [3] modified system (1.1) to allow the growth rate of the microorganism to depend upon the substrate concentration $\tau$ unit of time earlier. Their model is a system of two delay differential equations of the form

$$
\left\{\begin{array}{l}
x^{\prime}(t)=r x(t)[1-(x(t) / K)]-(x(t) y(t)) /\left(m^{2}+x^{2}(t)\right), \\
y^{\prime}(t)=y(t)\left[(\mu x(t-\tau)) /\left(m^{2}+x^{2}(t-\tau)\right)-d\right]
\end{array}\right.
$$

where $r, K, \mu, \tau$ and $d$ are positive constants and $m$ is a real constant. We remark that there are many different kinds of delayed predator-prey models in the literature, for more details we can refer to $[\mathbf{6}, \mathbf{1 0}, \mathbf{1 7}]$. For some systems with monotonic function response, we refer to $[\mathbf{9}, \mathbf{1 1}$, 16]. 
In the real world, the variation of the environment plays an important role in many biological and ecological systems. Thus, the assumption of periodicity of the parameters in the way (in a way) incorporates the periodicity of the environment (e.g., food supplies, mating habits, seasonal effects of weather, etc.). Motivated by such considerations, in the next section of this paper, we consider the existence of periodic solution of the corresponding nonautonomous periodic system with Monod-Haldane function response

$$
\left\{\begin{array}{l}
x^{\prime}(t)=x(t)[a(t)-b(t) x(t)]-(x(t) y(t)) /\left(m^{2}+c x(t)+x^{2}(t)\right), \\
y^{\prime}(t)=y(t)\left[(\mu(t) x(t-\tau)) /\left(m^{2}+c x(t-\tau)+x^{2}(t-\tau)\right)-d(t)\right],
\end{array}\right.
$$

where $x(t)$ and $y(t)$ represent predator and prey densities, respectively; $a(t)$ stands for the intrinsic growth rate of the prey population, $a(t) / b(t)$ stands for the carrying capacity, $\mu(t)$ stands for the rate of conversion of prey captured to predator and $d(t)$ stands for the natural death rate of the predators. They are all positive periodic continuous functions with period $\omega>0, c>0, m>0$ and $\tau \geq 0$ all constants. By using the coincidence degree theory developed by Gaines and Mawhin [8], we will establish the existence of at least one positive $\omega$-periodic solution of system (1.4). For work concerning the existence of periodic solutions of delay differential equations, we refer to $[\mathbf{7}, \mathbf{1 3}, \mathbf{1 5}, \mathbf{1 8}]$ and the references cited therein.

2. Existence of periodic solution. In order to obtain the existence of a positive periodic solution of system (1.4), we first make the following preparations.

Let $\Omega \subset R^{\prime \prime}$ be an open bounded set with closure $\bar{\Omega}$ and $f \in$ $C^{1}\left(\Omega, R^{n}\right) \cap C\left(\bar{\Omega}, R^{n}\right)$. For $x \in \Omega$, let $J_{f}(x)$ denote the Jacobian determinant of $f$ at $x$ and $S_{f}$ be the set of all critical points of $f$, i.e., $S_{f}=\left\{x \in \Omega: J_{f}(x)=0\right\}$. For $y \in R^{n} \backslash f\left(\partial \Omega \cup S_{f}\right)$, i.e., $y$ is a regular point of $f$, define the degree of $f$ at $y$ as

$$
\operatorname{deg}\{f, \Omega, y\}=\sum_{x \in f^{-1}(y)} \operatorname{sgn} J_{f}(x)
$$

with the agreement that $\sum_{\phi}=0$.

Let $X$ and $Z$ be two Banach spaces, Dom $L \subset X$ a subspace and $L: \operatorname{Dom} L \rightarrow Z$ a linear mapping. The kernel of $L$ is defined by 
$\operatorname{Ker} L=L^{-1}(0)$ and its range by $\operatorname{Im} L=L(\operatorname{Dom} L)$. Let Coker $L=$ $Z / \operatorname{Im} L$ be the quotient space of $Z$ under the equivalence relation $z^{\prime} \tilde{z}^{\prime} \Leftrightarrow z-z^{\prime} \in \operatorname{Im} L$. Thus, Coker $L=\{z+\operatorname{Im} L: z \in Z\}$. So $\operatorname{dim}$ Coker $L=\operatorname{codim} \operatorname{Im} L$.

The linear mapping $L$ is called a Fredholm mapping if (i) $\operatorname{Im} L$ is closed in $Z$ and (ii) $\operatorname{Ker} L$ and Coker $L$ are finitely dimensional. The index of $L$ is defined by

$$
\text { Ind } L=\operatorname{dim} \operatorname{ker} L-\operatorname{codim} \operatorname{Im} L \text {. }
$$

If Ind $L=0$, then $L$ is called a Fredholm mapping of index zero.

If $L$ is a Fredholm mapping of index zero, then there exist continuous projections $P: X \rightarrow X$ and $Q: Z \rightarrow Z$ such that

$$
\operatorname{Im} P=\operatorname{Ker} L \text { and } \operatorname{Im} L=\operatorname{Ker} Q=\operatorname{Im}(I-Q) .
$$

Define $L_{P}: \operatorname{Dom} L \cap \operatorname{Ker} P \rightarrow \operatorname{Im} L$ as the restriction $L_{\operatorname{Dom} L \cap \operatorname{Ker} P}$ of $L$ to Dom $L \cap \operatorname{Ker} P$. Then $L_{P}$ is an isomorphism. Define $K_{P}: \operatorname{Im} L \rightarrow$ Dom $L$ by

$$
K_{P}=L_{P}^{-1} \text {. }
$$

Then (a) $K_{P}$ is one-to-one and $P K_{P}=0$; (b) On $\operatorname{Im} L, L K_{P}=I$; (c) On Dom $L, K_{P} L=I-P$.

Let $N: X \rightarrow Z$ be a continuous mapping. $N$ is called $L$-compact on $\bar{\Omega}$ if $Q N(\bar{\Omega})$ is bounded and $K_{P}(I-Q) N: \bar{\Omega} \rightarrow X$ is compact. Since $\operatorname{Im} Q$ is isomorphic to $\operatorname{Ker} L$, there is an isomorphism

$$
J: \operatorname{Im} Q \longrightarrow \operatorname{Ker} L .
$$

Theorem A [2]. Let $X$ and $Z$ be two Banach spaces, and let $L$ be a Fredholm mapping of index zero. Suppose that $N: \bar{\Omega} \rightarrow Z$ is L-compact on $\bar{\Omega}$ with $\Omega$ open bounded in $X$. Furthermore, assume that

(a) for each $\lambda \in(0,1), x \in \partial \cap \operatorname{Dom} L$,

$$
L x \neq \lambda N x
$$

(b) for each $x \in \partial \Omega \cap \operatorname{Ker} L$,

$$
Q N_{x} \neq 0
$$


and

$\operatorname{deg}\{J Q N x, \Omega \cap \operatorname{Ker} L, 0\} \neq 0$, where $J Q N: \operatorname{Ker} L \rightarrow \operatorname{Ker} L$.

Then the equation $L x=N x$ has at least one solution in $\bar{\Omega} \cap \operatorname{Dom} L$.

In what follows we shall use the notation

$$
\bar{f}=\frac{1}{\omega} \int_{0}^{\omega} f(t) d t, \quad f^{L}=\min _{t \in[0, \omega]}|f(t)|, \quad f^{M}=\max _{t \in[0, \omega]}|f(t)|,
$$

where $f$ is a continuous periodic function with period $\omega$.

We are now in a position to state some lemmas which are useful in proving our main result.

Lemma 2.1. If system (1.4) has an w-periodic solution, then the following inequality holds:

$$
\bar{\mu} \geq(2 m+c) \bar{d} .
$$

The proof is obvious and we will omit it.

For the sake of convenience, in the rest of this section, we denote

$$
k=(\bar{\mu}-c \bar{d})^{2}-4(\bar{d} m)^{2} .
$$

Theorem 2.1. Assume that

$$
\bar{\mu}-c \bar{d}+\sqrt{k}>2 \bar{d} \bar{a} / \bar{b}, \bar{\mu}-c \bar{d}-\sqrt{k}<2 \bar{d} \bar{a} / \bar{b},
$$

and

$$
\bar{a} \mu^{L}>b^{M}\left(m^{2}+c \exp \left\{B^{*}\right\}+\exp \left\{2 B^{*}\right\}\right) \bar{d},
$$

hold true, where

$$
B^{*}=\ln \left(\begin{array}{l}
\bar{a} \\
\overline{\bar{b}}
\end{array}\right)+\bar{a} \omega
$$

Then system (1.4) has at least one positive $\omega$-periodic solution. 


\section{Proof. Since}

$$
\begin{aligned}
& x(t)=x(0) \exp \left\{\int_{0}^{t}\left[a(t)-b(t) x(t)-\frac{y(t)}{m^{2}+c x(t)+x^{2}(t)}\right] d t\right\}, \\
& y(t)=y(0) \exp \left\{\int_{0}^{t}\left[\frac{\mu(t) x(t-\tau)}{m^{2}+c x(t-\tau)+x^{2}(t-\tau)}-d(t)\right] d t\right\},
\end{aligned}
$$

the solution of system (1.4) remains positive for $t \geq 0$; let

$$
x(t)=\exp \left\{x_{1}(t)\right\}, \quad y(t)=\exp \left\{x_{2}(t)\right\},
$$

and derive that

$$
\begin{aligned}
x_{1}^{\prime}(t)= & a(t)-b(t) \exp \left\{x_{1}(t)\right\} \\
& -\frac{\exp \left\{x_{2}(t)\right\}}{m^{2}+c \exp \left\{x_{1}(t)\right\}+\exp \left\{2 x_{1}(t)\right\}}, \\
x_{2}^{\prime}(t)= & \frac{\mu(t) \exp \left\{x_{1}(t-\tau)\right\}}{m^{2}+c \exp \left\{x_{1}(t-\tau)\right\}+\exp \left\{2 x_{1}(t-\tau)\right\}}-d(t) .
\end{aligned}
$$

In order to use Theorem A to system (1.4), we take

$$
X=Z=\left\{x(t)=\left(x_{1}(t), x_{2}(t)\right)^{T} \in C\left(R, R^{2}\right): x(t+\omega)=x(t)\right\},
$$

and denote

$$
\|x\|=\left\|\left(x_{1}(t), x_{2}(t)\right)^{T}\right\|=\max _{t \in[0, \omega]}\left|x_{1}(t)\right|+\max _{t \in[0, \omega]}\left|x_{2}(t)\right| .
$$

Then $X$ and $Z$ are Banach spaces when they are endowed with the norms $\|\cdot\|$.

Set

$$
N x=\left[\begin{array}{c}
a(t)-b(t) \exp \left\{x_{1}(t)\right\}-\frac{\exp \left\{x_{2}(t)\right\}}{m^{2}+c \exp \left\{x_{1}(t)\right\}+\exp \left\{2 x_{1}(t)\right\}} \\
\frac{\mu(t) \exp \left\{x_{1}(t-\tau)\right\}}{m^{2}+c \exp \left\{x_{1}(t-\tau)\right\}+\exp \left\{2 x_{1}(t-\tau)\right\}}-d(t)
\end{array}\right]
$$

and

$$
L x=x^{\prime}, P x=\frac{1}{\omega} \int_{0}^{\omega} x(t) d t, x \in X, \quad Q z=\frac{1}{\omega} \int_{0}^{\omega} z(t) d t, z \in Z .
$$


Evidently, $\operatorname{Ker} L=\left\{x \mid x \in X, x=R^{2}\right\}, \operatorname{Im} L=\{z \mid z \in$ $\left.Z, \int_{0}^{\omega} z(t) d t=0\right\}$ is closed in $Z$ and $\operatorname{dim} \operatorname{Ker} L=\operatorname{codim} \operatorname{Im} L=2$. Hence, $L$ is a Fredholm mapping of index zero. Furthermore, the generalized inverse (to $L$ ) $K_{p}: \operatorname{Im} L-\operatorname{Ker} P \cap \operatorname{dom} L$ has the form

$$
K_{p}(z)=\int_{0}^{t} z(s) d s-\frac{1}{\omega} \int_{0}^{\omega} \int_{0}^{t} z(s) d s d t
$$

Thus,

$$
\begin{aligned}
& Q N x \\
& =\left[\begin{array}{c}
\frac{1}{\omega} \int_{0}^{\omega}\left[a(t)-b(t) \exp \left\{x_{1}(t)\right\}-\frac{\exp \left\{x_{2}(t)\right\}}{m^{2}+c \exp \left\{x_{1}(t)\right\}+\exp \left\{2 x_{1}(t)\right\}}\right] d t \\
\frac{1}{\omega} \int_{0}^{\omega}\left[\frac{\mu(t) \exp \left\{x_{1}(t-\tau)\right\}}{m^{2}+c \exp \left\{x_{1}(t-\tau)\right\}+\exp \left\{2 x_{1}(t-\tau)\right\}}-d(t)\right] d t
\end{array}\right],
\end{aligned}
$$

and

$$
\begin{aligned}
& K_{p}(I-Q) N \\
& =\left[\begin{array}{c}
\left.\int_{0}^{\omega}\left[a(s)-b(s) \exp \left\{x_{1}(s)\right\}-\frac{\exp \left\{x_{2}(s)\right\}}{m^{2}+c \exp \left\{x_{1}(t)\right\}+\exp \left\{2 x_{1}(s)\right\}}\right] d s\right] \\
\int_{0}^{t}\left[\frac{\mu(s) \exp \left\{x_{1}(s-\tau)\right\}}{m^{2}+c \exp \left\{x_{1}(s-\tau)\right\}+\exp \left\{2 x_{1}(s-\tau)\right\}}-d(s)\right] d s
\end{array}\right] \\
& -\left[\begin{array}{c}
\frac{1}{\omega} \int_{0}^{\omega} \int_{0}^{t}\left[a(s)-b(s) \exp \left\{x_{1}(s)\right\}\right. \\
\left.-\frac{\exp \left\{x_{2}(s)\right\}}{m^{2}+c \exp \left\{x_{1}(t)\right\}+\exp \left\{2 x_{1}(s)\right\}}\right] d s d t \\
\frac{1}{\omega} \int_{0}^{\omega} \int_{0}^{t}\left[\frac{\mu(s) \exp \left\{x_{1}(s-\tau)\right\}}{m^{2}+c \exp \left\{x_{1}(s-\tau)\right\}+\exp \left\{2 x_{1}(s-\tau)\right\}}-d(s)\right] d s d t
\end{array}\right] \\
& -\left[\begin{array}{c}
\left(\frac{t}{\omega}-\frac{1}{2}\right) \int_{0}^{\omega}\left[a(s)-b(s) \exp \left\{x_{1}(s)\right\}\right. \\
\left(\frac{t}{\omega}-\frac{1}{2}\right) \int_{0}^{\omega}\left[\frac{\exp \left\{x_{2}(s)\right\}}{m^{2}+c \exp \left\{x_{1}(t)\right\}+\exp \left\{2 x_{1}(s)\right\}}\right] d s
\end{array}\right]
\end{aligned}
$$

Clearly, $Q N$ and $K_{p}(I-Q) N$ are continuous and, moreover, $Q N(\bar{\Omega})$, $K_{p}(I-Q) N(\bar{\Omega})$ are relatively compact for any open bounded set $\Omega \subset X$. Hence, $N$ is $L$-compact on $\bar{\Omega}$; here $\Omega$ is any open bounded set in $X$. 
Now we reach the position to search for an appropriate open bounded subset $\Omega$ for the application of Theorem A. Corresponding to equation $L x=\lambda N x, \lambda \in(0,1)$, we have

$$
\left\{\begin{array}{l}
x_{1}^{\prime}(t)=\lambda\left[a(t)-b(t) \exp \left\{x_{1}(t)\right\}-\frac{\exp \left\{x_{2}(t)\right\}}{m^{2}+c \exp \left\{x_{1}(t)\right\}+\exp \left\{2 x_{1}(t)\right\}}\right] \\
x_{2}^{\prime}(t)=\lambda\left[\frac{\mu(t) \exp \left\{x_{1}(t-\tau)\right\}}{m^{2}+c \exp \left\{x_{1}(t-\tau)\right\}+\exp \left\{2 x_{1}(t-\tau)\right\}}-d(t)\right] d t=0 .
\end{array}\right.
$$

Suppose that $x(t)=\left(x_{1}, x_{2}\right) \in X$ is a solution of system (2.3) for a certain $\lambda \in(0,1)$. By integrating (2.3) over the interval [0, $\omega]$, we obtain

$$
\left\{\begin{array}{l}
\int_{0}^{\omega}\left[a(t)-b(t) \exp \left\{x_{1}(t)\right\}-\frac{\exp \left\{x_{2}(t)\right\}}{m^{2}+c \exp \left\{x_{1}(t)\right\}+\exp \left\{2 x_{1}(t)\right\}}\right] d t=0 \\
\int_{0}^{\omega}\left[\frac{\mu(t) \exp \left\{x_{1}(t-\tau)\right\}}{m^{2}+c \exp \left\{x_{1}(t-\tau)\right\}+\exp \left\{2 x_{1}(t-\tau)\right\}}-d(t)\right] d t=0
\end{array}\right.
$$

Hence,

$$
\int_{0}^{\omega}\left[b(t) \exp \left\{x_{1}(t)\right\}+\frac{\exp \left\{x_{2}(t)\right\}}{m^{2}+c \exp \left\{x_{1}(t)\right\}+\exp \left\{2 x_{1}(t)\right\}}\right] d t=\bar{a} \omega,
$$

and

$$
\int_{0}^{\omega}\left[\frac{\mu(t) \exp \left\{x_{1}(t-\tau)\right\}}{m^{2}+c \exp \left\{x_{1}(t-\tau)\right\}+\exp \left\{2 x_{1}(t-\tau)\right\}}\right] d t=\bar{d} \omega
$$

From (2.3), (2.4) and (2.5), we obtain

$$
\begin{aligned}
\int_{0}^{\omega}\left|x_{1}^{\prime}(t)\right| d t< & \int_{0}^{\omega}\left[b(t) \exp \left\{x_{1}(t)\right\}\right] d t \\
& +\int_{0}^{\omega}\left[\frac{\exp \left\{x_{2}(t)\right\}}{m^{2}+c \exp \left\{x_{1}(t)\right\}+\exp \left\{2 x_{1}(t)\right\}}\right] d t \\
& +\int_{0}^{\omega} a(t) d t \\
= & 2 \bar{a} \omega
\end{aligned}
$$


and

(2.7)

$$
\begin{aligned}
\int_{0}^{\omega}\left|x_{2}^{\prime}(t)\right| d t< & \int_{0}^{\omega}\left[\frac{\mu(t) \exp \left\{x_{1}(t-\tau)\right\}}{m^{2}+c \exp \left\{x_{1}(t-\tau)\right\}+\exp \left\{2 x_{1}(t-\tau)\right\}}\right] d t \\
& +\bar{d} \omega=2 \bar{d} \omega .
\end{aligned}
$$

Notice that $\left(x_{1}(t), x_{2}(t)\right)^{T} \in X$. Then there exist $\xi_{i}, \eta_{i} \in[0, \omega], i=1,2$, such that

$$
x_{i}\left(\xi_{i}\right)=\min _{t \in[0, \omega]} x_{i}(t), \quad x_{i}\left(\eta_{i}\right)=\max _{t \in[0, \omega]} x_{i}(t), \quad i=1,2 .
$$

By (2.4) and (2.8), we have

$$
\bar{a} \omega \geq \bar{b} \omega \exp \left\{x_{1}\left(\xi_{1}\right)\right\}
$$

that is,

$$
x_{1}\left(\xi_{1}\right) \leq \ln \left(\begin{array}{c}
\bar{a} \\
\bar{b}
\end{array}\right)
$$

From the first equation of (2.3), we have

$$
x_{1}^{\prime}(t) \leq \lambda a(t)<a(t),
$$

and

$$
-x_{1}^{\prime}(t)<b(t) \exp \left\{x_{1}(t)\right\}+\frac{\exp \left\{x_{2}(t)\right\}}{m^{2}+c \exp \left\{x_{1}(t)\right\}+\exp \left\{2 x_{1}(t)\right\}} .
$$

Then

$$
\int_{\xi_{1}}^{t} x_{1}(t) d t<\int_{\xi_{1}}^{t} a(t) d t, \quad \text { for } t \geq \xi_{1} .
$$

This implies that

$$
x_{1}(t)<\ln \left(\frac{\bar{a}}{\overline{\bar{b}}}\right)+\bar{a} \omega, \quad \text { for } t \geq \xi_{1},
$$

and

$$
\begin{aligned}
& \int_{t}^{\xi_{1}}\left(-x_{1}(t)\right) d t \\
& <\int_{t}^{\xi_{1}}\left[b(t) \exp \left\{x_{1}(t)\right\}+\frac{\exp \left\{x_{2}(t)\right\}}{m^{2}+c \exp \left\{x_{1}(t)\right\}+\exp \left\{2 x_{1}(t)\right\}}\right] d t \\
& \text { for } t \leq \xi_{1} .
\end{aligned}
$$


By (2.4), we obtain

$$
x_{1}(t)<\ln \left(\begin{array}{l}
\bar{a} \\
\bar{b}
\end{array}\right)+\bar{a} \omega, \quad \text { for } t \leq \xi_{1} .
$$

Hence,

$$
x_{1}(t)<\ln \left(\begin{array}{c}
\bar{a} \\
\overline{\bar{b}}
\end{array}\right)+\bar{a} \omega .
$$

By virtue of (2.5) and (2.8), we also have

$$
\frac{\bar{\mu} \exp \left\{x_{1}\left(\eta_{1}\right)\right\}}{m^{2}} \geq \bar{d} \omega
$$

and so

$$
x_{1}\left(\eta_{1}\right) \geq \ln \left(\frac{m^{2} \bar{d}}{\bar{\mu}}\right) .
$$

Then

$$
x_{1}(t) \geq x_{1}\left(\eta_{1}\right)-\int_{0}^{\omega}\left|x_{1}^{\prime}(t)\right| d t \geq \ln \left(\frac{m^{2} \bar{d}}{\bar{\mu}}\right)-2 \bar{a} \omega .
$$

It follows from (2.13) and (2.14) that

$$
\max _{t \in[0, \omega]}\left|x_{1}(t)\right| \leq \max \left\{\left|\ln \left(\begin{array}{c}
\bar{a} \\
\bar{b}
\end{array}\right)+2 \bar{a} \omega\right|,\left|\ln \left(\frac{m^{2} d}{\bar{\mu}}\right)-2 \bar{a} \omega\right|\right\}:=B_{1} .
$$

By (2.4), we have

$$
x_{2}\left(\xi_{2}\right) \leq \ln \left(\bar{a}\left(m^{2}+c \exp \left\{B^{*}\right\}+\exp \left\{2 B^{*}\right\}\right):=H_{1},\right.
$$

and by a similar analysis as above, we can obtain

$$
x_{2}(t)<H_{1}+\bar{d} \omega .
$$

From (2.5) we may conclude that

$$
\begin{gathered}
\int_{0}^{\omega}\left[\frac{\mu(t) \exp \left\{x_{1}(t-\tau)\right\}}{m^{2}+c \exp \left\{x_{1}(t-\tau)\right\}+\exp \left\{2 x_{1}(t-\tau)\right\}}\right] d t \\
\geq \frac{\mu^{L}}{m^{2}+c \exp \left\{B^{*}\right\}+\exp \left\{2 B^{*}\right\}} \int_{0}^{\omega} \exp \left\{x_{1}(t-\tau)\right\} d t \\
=\frac{\mu^{L}}{m^{2}+c \exp \left\{B^{*}\right\}+\exp \left\{2 B^{*}\right\}} \int_{0}^{\omega} \exp \left\{x_{1}(t)\right\} d t
\end{gathered}
$$


which implies that

$$
\int_{0}^{\omega} \exp \left\{x_{1}(t)\right\} d t \leq \frac{\left(m^{2}+c \exp \left\{B^{*}\right\}+\exp \left\{2 B^{*}\right\}\right) \bar{d} \omega}{\mu^{L}} .
$$

Notice that

$$
\int_{0}^{\omega}\left[\frac{\exp \left\{x_{2}(t)\right\}}{m^{2}+c \exp \left\{x_{1}(t)\right\}+\exp \left\{2 x_{1}(t)\right\}}\right] d t \leq \frac{1}{m^{2}} \int_{0}^{\omega} \exp \left\{x_{2}(t)\right\} d t
$$

and

$$
\begin{aligned}
\int_{0}^{\omega} b(t) \exp \left\{x_{1}(t)\right\} d t & \leq b^{M} \int_{0}^{\omega} \exp \left\{x_{1}(t)\right\} d t \\
& \leq \frac{b^{M}\left(m^{2}+c \exp \left\{B^{*}\right\}+\exp \left\{2 B^{*}\right\}\right) d \omega}{\mu^{L}}
\end{aligned}
$$

also in view of (H2) and (2.4), we have

$$
\begin{aligned}
\int_{0}^{\omega} \exp \left\{x_{2}(t)\right\} d t & \leq \frac{\bar{a} \mu^{L}-b^{M}\left(m^{2}+c \exp \left\{B^{1}\right\}+\exp \left\{2 B_{1}\right\}\right) \bar{d}}{\mu^{L}} m^{2} \omega \\
& :=\exp \left\{H_{2}\right\} \omega
\end{aligned}
$$

which implies that

$$
x_{2}\left(\eta_{2}\right) \geq H_{2}
$$

and so

$$
x_{2}(t) \geq x_{2}\left(\eta_{2}\right)-\int_{0}^{\omega}\left|x_{2}^{\prime}(t)\right| d t \geq H_{2}-2 \bar{d} \omega .
$$

Thus,

$$
\max _{t \in[0, \omega]}\left|x_{2}(t)\right| \leq \max \left\{\left|H_{1}+\bar{d} \omega\right|,\left|H_{2}-2 \bar{d} \omega\right|\right\}:=B_{2}
$$

Clearly, $B_{i}, i=1,2$, are independent of $\lambda$. Under the assumptions in Theorem 2.1, it is easy to show that the system of algebraic equations

$$
\left\{\begin{array}{l}
\bar{a}-\bar{b} u-v /\left(m^{2}+c u+u^{2}\right)=0 \\
\bar{d}-(\bar{\mu} u) /\left(m^{2}+c u+u^{2}\right)=0
\end{array}\right.
$$


has a unique solution $\left(u^{*}, v^{*}\right)^{T} \in \operatorname{int} R_{+}^{2}$ with $u^{*}, v^{*}>0$. Denote

$$
B=B_{1}+B_{2}+B_{3},
$$

where $B_{3}>0$ is taken sufficiently large such that

$$
\left\|\left(\ln \left\{v^{*}\right\}, \ln \left\{u^{*}\right\}\right)\right\|=\left|\ln \left\{v^{*}\right\}\right|+\left|\ln \left\{u^{*}\right\}\right|<B_{3},
$$

and define

$$
\Omega=\{x(t) \in X:\|x\|<B\} .
$$

It is clear that $\Omega$ satisfies condition (a) of Theorem A. When

$$
x=\left(x_{1}, x_{2}\right)^{T} \in \partial \Omega \cap \operatorname{Ker} L=\partial \Omega \cap R^{2},
$$

$x$ is a constant vector in $R^{2}$ with $\|x\|=B$. Then

$$
Q N_{x}=\left[\begin{array}{c}
\bar{a}-\bar{b} \exp \left\{x_{1}\right\}-\frac{\exp \left\{x_{2}\right\}}{m^{2}+c \exp \left\{x_{1}\right\}+\exp \left\{2 x_{1}\right\}} \\
-\bar{d}+\frac{\mu \exp \left\{x_{1}\right\}}{m^{2}+c \exp \left\{x_{1}\right\}+\exp \left\{2 x_{1}\right\}}
\end{array}\right]=0 .
$$

Furthermore, let $J: \operatorname{Im} Q \rightarrow \operatorname{Ker} L, x \rightarrow x$; in view of the assumptions in Theorem 2.1, it is easy to see

$$
\operatorname{deg}\{J Q N x, \Omega \cap \operatorname{Ker} L, 0\} \neq 0 .
$$

By now we know that $\Omega$ verifies all the requirements of Theorem A and then system (2.2) has at least one $\omega$-periodic solution. By the medium of (2.1), we derive that (1.4) has at least one positive $\omega$-periodic solution. The proof is complete.

Remarks. A recent paper [15] discussed a periodic predator-prey system with a type IV functional response, a sufficient condition for the system has at least two positive periodic solutions was given. When we propose a model

$$
\left\{\begin{aligned}
x^{\prime}(t)=x(t)\left[\frac{1}{2}-\frac{1}{3} \cos (\pi t)-\left(\frac{2}{3}+\frac{1}{3} \sin (\pi t)\right) x(t)\right] & -\frac{x(t) y(t)}{1+x(t)+x^{2}(t)} \\
y^{\prime}(t)=y(t)\left[\frac{(26-\cos (2 \pi t)) x(t-1)}{1+x(t-1)+x^{2}(t-1)}\right. & \left.-\left(1+\frac{1}{2} \sin (\pi t)\right)\right] .
\end{aligned}\right.
$$




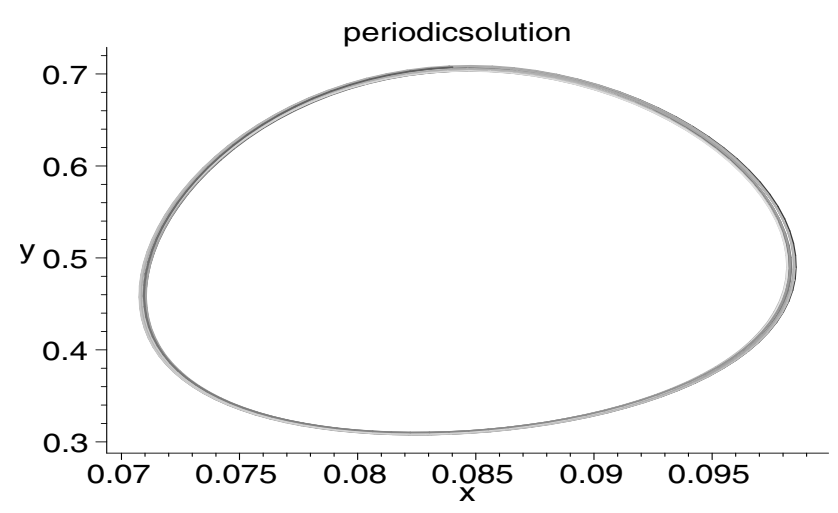

FIGURE 1. $x(0)=0.08401, y(0)=0.705$ and stepsize $=0.002$.

We should point out that this system does not satisfy the condition of Chen's, while the sufficient condition we conclude here is actionable. It is easy to verify that all the conditions in Theorem 2.1 hold true. Then the above equation has at least one positive solution of period 1 . We sketch the periodic solution in Figure 1.

3. Discussion. The existence of the positive periodic solution for system (1.4) in biology indicates that, under some reasonable conditions, the prey species and the predator species will coexist in the long run. In fact, conditions (H0) and (H1) imply the existence and uniqueness of positive equilibrium for the following system

$$
\left\{\begin{array}{l}
x^{\prime}(t)=x(t)[\bar{a}-\bar{b} x(t)]-\frac{x(t) y(t)}{m^{2}+c x(t)+x^{2}(t)} \\
y^{\prime}(t)=y(t)\left[\frac{\bar{\mu} x(t-\tau)}{m^{2}+c x(t-\tau)+x^{2}(t-\tau)}-\bar{d}\right]
\end{array}\right.
$$

Furthermore, condition (H2) along with (H0) and (H1) assure the existence of positive periodic solutions for system (1.4). And our result show that the common period $\omega$ for the coefficient functions (i.e., the intrinsic growth rate of the prey population, the rate of conversion of prey captured to predator and the natural death rate of the predators etc.) cannot be very large; enumeration examples show that when their 
common period is large enough, the positive periodic solution of the system will disappear. Therefore we conclude that there must be a critical value for this common period. What should this value be? This will be an interesting problem for us to study later.

\section{REFERENCES}

1. J.F. Andrews, A mathematical model for the continuous culture of microorganisms utilizing inhibitory substrates, Biotech. Bioengineering 10 (1986), 707-723.

2. A.A. Berryman, The origins and evolution of predator-prey theory, Ecology 75 (1992), 1530-1535.

3. A.W. Bush and A.E. Cook, The effect of time delay and growth rate inhibition in the bacterial treatment of wastewater, J. Theory Biol. 63 (1976), 385-395.

4. G.J. Butler and G.S.K. Wolkowicz, A mathematical model of the chemostat with a general class of functions describing nutrient uptake, SIAM J. Appl. Math. 45 (1985), 138-151.

5. Y. M. Chen, Multiple periodic solutions of delayed predator-prey systems with type IV functional responses, Nonlinear Anal. 5 (2004), 45-53.

6. H.I. Freedman, Deterministic mathematical models in population ecology, Marcel Dekker, New York, 1980.

7. H.I. Freedman and J. Wu, Periodic solutions of single-species models with periodic delay, SIAM J. Math. Anal. 23 (1992), 689-701.

8. R.E. Gaines and J.L. Mawhin, Coincidence degree and nonlinear differential equations, Springer, Berlin, 1977.

9. H.F. Huo, W.T. Li and S.S. Cheng, Periodic solutions of two-species diffusive models with continuous time delays, Demonst. Math. 35 (2002), 433-466.

10. Y. Kuang, Delay differential equations with applications in population dynamics, Academic Press, New York, 1993.

11. Y.K. Li and Y. Kuang, Periodic solutions of periodic delay Lotka-Volterra equations and systems, J. Math. Anal. Appl. 255 (2001), 260-280.

12. S.G. Ruan and D.M. Xiao, Global analysis in a predator-prey system with non-monotonic functional response, SIAM J. Appl. Math. 61 (2001), 1445-1472.

13. H.L. Smith and Y. Kuang, Periodic solutions of delay differential equations of threshold-type delay, in Oscillation and dynamicals in delay equations, Graef and Hale, eds., Contemporary Math. 129, American Mathematical Society, Providence, 1992.

14. W. Sokol and J.A. Howell, Kinetics of phenol oxidation by washed cells, Biotechnol. Bioengineering 23 (1980), 2039-2049.

15. B.R. Tang and Y. Kuang, Existence, uniqueness and asymptotic stability of periodic solutions of periodic functional differential systems, Tohoku Math. J. 49 (1997), 217-239.

16. L.L. Wang and W.T. Li, Existence and global stability of positive periodic solutions of a predator-prey system with delays, Appl. Math. Comput. 146 (2003), 167-185. 
17. L.L. Wang and W.T. Li, Periodic solutions and permanence for a delayed nonautonomous ratio-dependent predator-prey model with Holling type functional response, J. Comput. Appl. Math. 162 (2004), 341-357.

18. T. Zhao, Y. Kuang and H.L. Smith, Global existence of periodic solutions in a class of delayed Gause-type predator-prey systems, Nonlinear Anal. 28 (1997), 1373-1394.

19. H.P. Zhu, S.A. Campbell and G.S.K. Wolkowicz, Bifurcation analysis of a predator-prey system with nonmonotonic functional response, SIAM J. Appl. Math. 63 (2002), 636-682.

School of Mathematics and Information, Ludong University, Yantai, Shandong 264025, P.R. China and Department of Mathematics, Beijing Institute of Technology, Beijing 100081, P.R. China

Email address: wangll_1994@sina.com, llwang@bit.edu.cn

School of Mathematics and Information, Ludong University, Yantai, Shandong 264025, P.R. China and Department of Mathematics, Southeast University, Nanjing, Jiangsu, 210096, P.R. China

Email address: fanyh_1993@sina.com

Department of Applied Mathematics, Beijing Institute of Technology, BeiJing 100081, P.R. China 